\title{
Pathotypic characterization of Newcastle disease virus isolated from vaccinated chicken in West Java, Indonesia
}

\author{
Dwi Desmiyeni Putri ${ }^{1,2}$, Ekowati Handharyani ${ }^{3}$, Retno Damajanti Soejoedono ${ }^{4}$, Agus Setiyono ${ }^{3}$, Ni Luh Putu Ika Mayasari ${ }^{4}$ \\ and Okti Nadia Poetri ${ }^{4}$ \\ 1. Study Program of Animal Biomedical Science, IPB Graduate School, Bogor Agricultural University, Bogor, Indonesia; \\ 2. Study Program of Animal Husbandry, Department of Animal Husbandry, State Polytechnic of Lampung, Lampung, \\ Indonesia; 3. Department of Veterinary Clinic Reproduction and Pathology, Faculty of Veterinary Medicine, Bogor \\ Agricultural University, Bogor, Indonesia; 4. Department of Animal Diseases and Veterinary Public Health, Faculty of \\ Veterinary Medicine, Bogor Agricultural University, Bogor, Indonesia. \\ Corresponding author: Dwi Desmiyeni Putri, e-mail: desmiyenidwi@polinela.ac.id, \\ Co-authors: EH: ekowatieko@apps.ipb.ac.id, RDS: retnodmail@yahoo.com, AS: agusetiyo@yahoo.com, \\ NLPIM: mayasari.ika@gmail.com, ONP: diapoetri@gmail.com \\ Received: 21-10-2016, Accepted: 09-03-2017, Published online: 22-04-2017
}

doi: 10.14202/vetworld.2017.438-444 How to cite this article: Putri DD, Handharyani E, Soejoedono RD, Setiyono A, Mayasari NI, Poetri ON (2017) Pathotypic characterization of Newcastle disease virus isolated from vaccinated chicken in West Java, Indonesia, Veterinary World, 10(4): 438-444.

\begin{abstract}
Aim: This research was conducted to differentiate and characterize eight Newcastle disease virus (NDV) isolates collected from vaccinated chicken at commercial flocks in West Java, Indonesia, in 2011, 2014 and 2015 by pathotype specific primers.

Materials and Methods: A total of eight NDV isolates collected from clinical outbreaks among commercial vaccinated flocks in West Java, Indonesia, in 2011, 2014, and 2015 were used in this study. Reverse transcription-polymerase chain reaction was used to detect and differentiate virulence of NDV strains, using three sets of primers targeting their $\mathrm{M}$ and $\mathrm{F}$ gene. First primers were universal primers to detect NDV targeting matrix (M) gene. Other two sets of primers were specific for the fusion (F) gene cleavage site sequence of virulent and avirulent NDV strains.

Results: Our results showed that three isolates belong to NDV virulent strains, and other five isolates belong to NDV avirulent strains. The nucleotide sequence of the F protein cleavage site showed ${ }^{112} \mathrm{~K} / \mathrm{R}-\mathrm{R}-\mathrm{Q} / \mathrm{R}-\mathrm{K}-\mathrm{R} / \mathrm{G}-\mathrm{F}^{117}$ on $\mathrm{NDV}$ virulent strains and ${ }^{112} \mathrm{G}-\mathrm{K} / \mathrm{R}-\mathrm{Q}-\mathrm{G}-\mathrm{R}-\mathrm{L}^{117}$ on NDV avirulent strain.

Conclusion: Result from the current study suggested that NDV virulent strain were circulating among vaccinated chickens in West Java, Indonesia; this might possess a risk of causing ND outbreaks and causing economic losses within the poultry industry.
\end{abstract}

Keywords: F gene, M gene, molecular pathotyping, Newcastle disease virus, vaccinated chickens, virulence.

\section{Introduction}

Newcastle disease (ND) is a highly contagious and fatal disease of poultry [1], due to the potential for devasting losses [2] and categorized in list A disease by the Office International des Epizooties (OIE) [3-5]. The diseases were caused by ND virus (NDV) or Avian Paramyxovirus Type 1, which belongs to the genus Avulavirus in the family of Paramyxoviridae [6]. NDV infection would lead to a broad range of clinical signs, such as asymptomatic enteric to systemic infection with up to $100 \%$ mortality [7]. Based on the severity of the disease in chickens, NDV is categorized into asymptomatic enteric (avirulence), lentogenic (low virulence), mesogenic (intermediate virulence), and velogenic (high virulence) pathotypes [6]. The velogenic and mesogenic (virulent) strains have been

Copyright: Putri, et al. Open Access. This article is distributed under the terms of the Creative Commons Attribution 4.0 International License (http://creativecommons.org/licenses/by/4.0/), which permits unrestricted use, distribution, and reproduction in any medium, provided you give appropriate credit to the original author(s) and the source, provide a link to the Creative Commons license, and indicate if changes were made. The Creative Commons Public Domain Dedication waiver (http://creativecommons.org/ publicdomain/zero/1.0/) applies to the data made available in this article, unless otherwise stated. identified as the causative agent of ND outbreaks in many countries. The avirulent strains have been used as live vaccines to control the disease; however, ND outbreaks have been reported in vaccinated poultry consistenly [4].

In general, NDV characterization was determined using virus isolation followed by in vivo tests such as intracerebral pathogenicity index (ICPI), intravenous pathogenicity index (IVPI), and mean death time (MDT) in specified pathogen free (SPF) chicken embryo/birds for pathotyping $[3,8]$. However, molecular methods - such as reverse transcription-polymerase chain reaction (RT-PCR) and amino acid sequencing - were developed for NDV detection and pathotyping $[4,9,10]$. The previous study has improved RT-PCR using pathotype specific primers to detect and differentiate virulence of NDV strains; this technique seems to be more efficient for NDV pathotyping $[11,12]$. The pathotype specific primers set was conducted based on nucleotides sequence at the $\mathrm{F}$ gene cleavage site, which has been shown to be a major determinant of NDV virulence [13-15].

Indonesia has a large poultry population, and West Java is the province with the largest poultry 
population i.e. $497,814,132$ or $44.64 \%$ from national poultry population [16]. West Java has experienced with recurrent of NDV outbreak among vaccinated commercial flocks [17]. The previous study reported that NDV genotype VII virulent strain was causing the outbreaks among backyards and commercials flock since 2009 [17,18]. NDV genotype VII was also isolated from live bird market in some district in West Java, such as Bogor, Sukabumi, and Tangerang [19]. Understanding the pathotypic character of NDV isolated from clinical outbreaks were important to control the diseases in West Java; however, such information is limited. Therefore, this study was designed to differentiate and characterize NDV isolates collected from clinical outbreaks among commercial vaccinated flocks in West Java, Indonesia, in 2011, 2014, and 2015 by pathotype specific primers. The virulence of NDVs was evaluated by molecular analyses of the nucleotide and deduced amino acid sequences of the $\mathrm{F}$ gene. Our results might provide a better understanding of NDV circulating in the region and contribute for controlling the diseases.

\section{Materials and Methods}

\section{Ethical approval}

This study were performed according to the regulations for Research in Animal Health of Indonesian Law on Livestock and Animal Health (UU/18/2009, article 80).

\section{Virus \\ NDV isolates}

A total of eight NDV isolates collected from clinical outbreaks among commercial vaccinated flocks in West Java, Indonesia, in 2011, 2014, and 2015 were used in this study. Origin and year of isolation of NDV isolates were shown in Table-1. These isolates are $\mathrm{NDV} / \mathrm{Ck} / \mathrm{BGR} / 11, \mathrm{NDV} / \mathrm{Ck} / \mathrm{GS} / 14, \mathrm{NDV} / \mathrm{Ck} / /$ $\mathrm{JP} / 14, \mathrm{NDV} / \mathrm{Ck} / \mathrm{LG} / 15, \mathrm{NDV} / \mathrm{Ck} / \mathrm{CJR} / 15, \mathrm{NDV} / \mathrm{Ck} /$ $\mathrm{BGR} / 15, \mathrm{NDV} / \mathrm{Ck} / \mathrm{TRG} / 15$, and NDV/Ck/LWG/15. Two NDV strains representing different pathotypes: NDV/Lasota (avirulent) and NDV/Sato (virulent) were used as representative strains. Before testing in RT-PCR, all the samples were propagated once into SPF embryonated chicken eggs (ECEs) and allantoic fluid were used for further studies.

\section{$R N A$ isolation}

RNAs of the viruses were extracted from allantoic fluids using QIAamp $^{\circledR}$ Viral RNA Mini Kit catalog number 52904 (Qiagen, Germany) according to manufacturer instruction [20]. $140 \mu \mathrm{l}$ of sample suspension was used for extraction, and RNA was diluted in a final volume $60 \mu 1$ and store at $-80^{\circ} \mathrm{C}$.

\section{RT-PCR}

Amplification

RT-PCR was performed using one-step RT-PCR kit (Qiagen, Germany) according to manufacturer instruction. RT-PCR reaction mixture of each sample consisted of $2 \mu 1$ of dNTPs mix (10 mM), $2 \mu 1$ of forward primer $(10 \mathrm{pM}), 2 \mu 1$ of reverse primer $(10 \mathrm{pM})$, $2 \mu 1$ of purified template RNA, $10 \mu 1$ of $5 \times$ Onestep RT-PCR Qiagen buffer, $30 \mu 1$ of RNase-free water, and $2 \mu 1$ one-step RT-PCR enzyme mixed in a final volume of $50 \mu 1$. Amplification for $\mathrm{M}$ gene was setup as $45^{\circ} \mathrm{C}$ for $60 \mathrm{~min}$ followed by initial denaturation at $95^{\circ} \mathrm{C}$ for $5 \mathrm{~min}$ and 35 cycles of denaturation at $95^{\circ} \mathrm{C}$ for $30 \mathrm{~s}$, annealing at $50^{\circ} \mathrm{C}$ for $30 \mathrm{~s}$, extension at $72^{\circ} \mathrm{C}$ for $40 \mathrm{~s}$ and final extension at $72^{\circ} \mathrm{C}$ for $10 \mathrm{~min}$. Amplification for $\mathrm{F}$ gene were performed according to the following protocol: $45^{\circ} \mathrm{C}$ for $60 \mathrm{~min}$ for c-DNA synthesis followed by initial denaturation at $94^{\circ} \mathrm{C}$ for $5 \mathrm{~min}$, and 35 cycles of $94^{\circ} \mathrm{C}$ for $1 \mathrm{~min}, 50^{\circ} \mathrm{C}$ for $1 \mathrm{~min}, 72^{\circ} \mathrm{C}$ for $1 \mathrm{~min}$, with a final elongation step of $5 \mathrm{~min}$ for $72^{\circ} \mathrm{C}$.

\section{Primers}

Three sets of primers were used for amplification. First primers set are NDV-MF/MR were universal primers targeting for $\mathrm{M}$ gene. Another two set of primers targeting $\mathrm{F}$ gene cleavage site: NDV-FA/FB were specific for virulent NDV and NDV-FA/FC were specific for avirulent NDV strains [11]. Nucleotide sequences of all primers were presented in Table-2.

\section{Electrophoresis}

PCR products were separated in $1.5 \%$ agarose gel in $1 \times$ Tris-acetate-ethylenediaminetetraacetic acid buffer stained with ethidium bromide, compared with molecular mass marker and visualized by ultraviolet transillumination.

\section{Nucleotide sequencing}

Sequencing of PCR product

The positive results of PCR products were sequenced using BigDye ${ }^{\circledR}$ Terminator v3.1 cycle Sequencing Kit (Thermo Fisher Scientific, USA) according to manufacturer instruction. The first stage began with the purification of PCR products using Centricon ${ }^{\circledR}-100$ columns (Millipore, USA). Purified PCR products were sequenced by First Base Company (Malaysia) with the primer NDV-FA. The nucleotide sequencing was performed according to the following protocol: denaturation at $96^{\circ} \mathrm{C}$ for $1 \mathrm{~min}$, followed by 25 cycles of $96^{\circ} \mathrm{C}$ for $10 \mathrm{~s}, 50^{\circ} \mathrm{C}$ for $5 \mathrm{~s}$, and $60^{\circ} \mathrm{C}$ for $4 \mathrm{~min}$, with a final elongation step of $5 \mathrm{~min}$ for $72^{\circ} \mathrm{C}$. The final stage was the purification of the product cycle sequencing using Centri-Sep ${ }^{\mathrm{TM}}$ spin columns (Thermo Fisher Scientific, USA) according to manufacturer instruction.

\section{Analysis of nucleotide sequence}

The obtained sequence was edited using BioEdit Sequence Alignment Editor Version 7.0.9.0. Alignment of the sequences was performed using MEGA version 6 [21].

\section{Results}

\section{$M$ and $F$ gene RT-PCR}

$M$ gene amplification results are presented in Figure-1 and Table-1. All isolates showed specific 
Table-1: Data and characteristics of the NDV isolates used for the investigation.

\begin{tabular}{|c|c|c|c|c|c|c|c|}
\hline \multirow[t]{2}{*}{ Isolate $^{a}$} & \multirow[t]{2}{*}{ Originb } & \multirow[t]{2}{*}{ Year of isolation } & \multicolumn{3}{|c|}{ RT-PCR resultc } & \multirow[t]{2}{*}{ Cleavage site sequence } & \multirow[t]{2}{*}{ Pathotype } \\
\hline & & & $\mathbf{M}$ & FA/FB & FA/FC & & \\
\hline NDV/Ck/BGR/11 & Bogor & 2011 & + & + & - & R-R-Q-K-R-F & Virulent \\
\hline NDV/Ck/GS/14 & Gunung Sindur & 2014 & + & + & - & K-R-R-K-R-F & Virulent \\
\hline NDV/Ck/JP/14 & Jampang & 2014 & + & - & + & G-K-Q-G-R-L & Avirulent \\
\hline NDV/Ck/LG/15 & Legok & 2015 & + & - & + & G-K-Q-G-R-L & Avirulent \\
\hline NDV/Ck/CJR/15 & Cianjur & 2015 & + & - & + & G-R-Q-G-R-L & Avirulent \\
\hline NDV/Ck/BGR/15 & Bogor & 2015 & + & - & + & G-R-Q-G-R-L & Avirulent \\
\hline NDV/Ck/TRG/15 & Tangerang & 2015 & + & - & + & G-K-Q-G-R-L & Avirulent \\
\hline NDV/Ck/LWG/15 & Leuwiliang & 2015 & + & + & - & R-R-Q-K-G-F & Virulent \\
\hline
\end{tabular}

${ }^{a}$ All isolates were collected from vaccinated chickens showing clinical sign of ND, bAll origin were located at West Java Province, $c M=$ Matriks gene (universal primer for detecting NDV); FA/FB=F gene (virulent NDV); FA/FC=F gene (avirulent NDV), Lanes 9-10 are Sato and 11-12 are Lasota (used as positive control). NDV=Newcastle disease virus, RT-PCR $=$ Reverse transcription-polymerase chain reaction

Table-2: Matrix (M) and Fusion (F) primers sequence used for the investigation.

\begin{tabular}{lllr}
\hline Gene & Code & Sequence & Position (bp) \\
\hline Matrix & MF & 5'-TCGAGTCTGTACAATCTTGC-3' & 232 \\
Matrix & MR & 5'-GTCCGAGCACATCACTGAGC-3' & \\
Fusion & FA & 5'-TTGATGGCAGGCCTCTTGC-3' & $141-159$ \\
Fusion & FB & $5^{\prime}$-AGCGT (C/T) TCTGTCTCCT-3' & $395-380$ \\
Fusion & FC & $5^{\prime}-$ G (A/G) CG (A/T) CCCTGT (C/T) TCCC-3' & $395-380$ \\
\hline
\end{tabular}

band at $232 \mathrm{bp}$ indicating that all isolates were NDV. Identification of NDV virulent and avirulent strain was determined using combination of $\mathrm{F}$ gene primer (pathotype specific primers) developed by Kant et al. [11]. F gene amplification results are presented in Figures-2 and 3; Table-1. Three isolates could be amplified using NDV-FA/FB primer, while other five isolates could be amplified using NDV-FA/FC primer. Based on $\mathrm{F}$ gene amplification result, three isolates which is $\mathrm{NDV} / \mathrm{Ck} / \mathrm{BGR} / 11, \mathrm{NDV} / \mathrm{Ck} / \mathrm{GS} / 14$, and NDV/ $\mathrm{Ck} / \mathrm{LWG} / 15$ were belong to virulent NDV strains, while other five isolates: NDV/Ck/JP/14, NDV/Ck/LG/15, $\mathrm{NDV} / \mathrm{Ck} / \mathrm{CJR} / 15, \mathrm{NDV} / \mathrm{Ck} / \mathrm{BGR} / 15$, and $\mathrm{NDV} / \mathrm{Ck} /$ TRG/15 were belong to avirulent NDV strains.

\section{Cleavage site sequencing}

Nucleotide sequence of $\mathrm{F}$ gene cleavage site is presented in Figure-4. NDV virulent strains have multiple basic amino acid arginine $(\mathrm{R})$ or lysine $(\mathrm{K})$ at the fusion $(\mathrm{F})$ cleavage site at residues $112-113$ and $115-116$ and showed motif ${ }^{112} \mathrm{~K} / \mathrm{R}-\mathrm{R}-\mathrm{Q} / \mathrm{R}-\mathrm{K}-\mathrm{R} / \mathrm{G}-\mathrm{F}^{117}$. NDV/Ck/BGR/11 showed motif R-R-G-K-R-F, NDV/ $\mathrm{Ck} / \mathrm{GS} / 14$ showed motif K-R-R-K-R-F, and NDV/Ck/ LWG/15 showed motif R-R-Q-K-G-F on its F gene cleavage site sequence. NDV/Ck/LWG/15 has different pattern with other two virulent isolates; however, the sequence homology with $\mathrm{NDV} / \mathrm{Ck} / \mathrm{GS} / 14$ were $96 \%$ (with the nucleotide in position 335,341 , and 346 were different). NDV avirulent strains shown motif ${ }^{112} \mathrm{G}-\mathrm{K} / \mathrm{R}-\mathrm{Q}-\mathrm{G}-\mathrm{R}-\mathrm{L}^{117}$ on its cleavage site sequence. $\mathrm{NDV} / \mathrm{Ck} / \mathrm{JP} / 14, \quad \mathrm{NDV} / \mathrm{Ck} / \mathrm{LG} / 15$, and $\mathrm{NDV} / \mathrm{Ck} /$ TGR/15 have motif G-K-Q-G-R-L and the NDV/Ck/ $\mathrm{JP} / 14$ and NDV/Ck/LG/14 shown motif G-R-Q-G$\mathrm{R}-\mathrm{L}$. Cleavage site nucleotide sequence results were in accordance with amplification $\mathrm{F}$ gene using pathotype specific primer results (Figure-4).

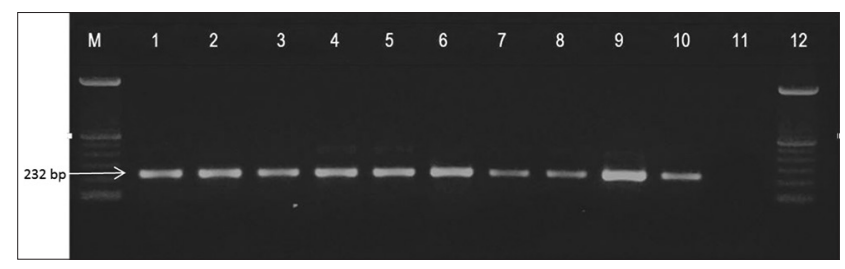

Figure-1: Matrix gene amplification results. Polymerase chain reaction product size of $232 \mathrm{bp}$. The amplicons were electrophoresed in 1,5\% agarose gel. Lanes: $M$ - Molecular size marker; Lanes 1-8 are Newcastle disease virus (NDV) field isolates, Lanes $9 \mathrm{NDV} /$ Sato and 10 are NDV/Lasota (used as positive control); Lane 11 is non template control.

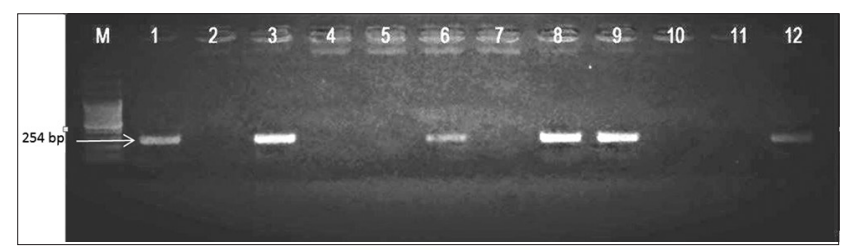

Figure-2: Fusion gene amplification results. Reverse transcription-polymerase chain reaction amplification of the Newcastle disease virus fusion gene using FA (forward) and FB, FC (reverse) primer combination, which gave a product size of $254 \mathrm{bp}$. The amplicons were electrophoresed in $1.5 \%$ agarose gel. Lanes: $M$ - Molecular size marker; Lanes $1,3,5,7,9,11$ (FA/FB), lanes 2, 4, 6, 8, 10, 12 (FA/FC), Lane 1-2 - Newcastle disease virus (NDV)/Ck/BGR/11, 3-4 - NDV/Ck/GS/14, 5-6 - NDV/Ck/JP/14, 7-8 - NDV/Ck/ LG/15, Lanes $9-10$ are Sato and 11-12 are Lasota (used as positive control).

\section{Discussion}

Indonesia has been an endemic country for ND, despite control strategies that have been done to eradicate the viruses. In general, ND vaccination program has been applied among commercial poultry flocks in Indonesia. However, in 2009 and 2010, clinical ND outbreaks among vaccinated commercial flocks 
were reported, causing up to $70-80 \%$ mortality [18]. Diseases control program need better understanding of circulating viruses characteristic, and this could be achieved by routine surveillance. Diagnosis of diseases among poultry flocks should be performed quickly and accurate.

According to OIE [3], the gold standard methods NDV laboratory diagnosis are virus isolation using ECE aged 9-11 days. NDV pathogenicity was assessed by determining the MDT of embryos, ICPI,

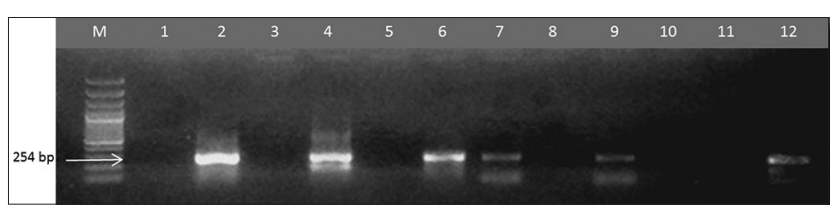

Figure-3: Reverse transcription-polymerase chain reaction amplification of the Newcastle disease virus fusion gene using FA (forward) and FB, FC 2 (reverse) primer combination, which gave a product size of $254 \mathrm{bp}$. The amplicons were electrophoresed in $1.5 \%$ agarose gel. Lanes: $\mathrm{M}$ - molecular size marker; Lanes 1, 3, 5, 7, 9, 11 (FA/FB), Lanes 2, 4, $6,8,10,12$ (FA/FC), Lane 1-2- Newcastle disease virus (NDV)/Ck/CJR/15, 3-4- NDV/Ck/BGR/15, 5-6- NDV/Ck/ TRG/15, 7-8 NDV/Ck/LWG/15. Lanes 9-10 are Sato and 11-12 are Lasota (used as positive control). and IVPI [4]. However, this method requires a relatively long time for NDV diagnosis, molecular technique such RT-PCR offered faster result [4,22,23].

RT-PCR for NDV detection was first described by Jestin and Jestin [22], and this technique has been modified by Seal et al. [24], Aldous and Alexander [4], Rabalski et al. [25] using universal primers to detect all NDVs; Kant et al. [11], Lai et al. [26], and Al-Shammari et al. [27] developed pathotype specific primers that enable rapid differentiation of the pathotype; also Kho et al. [23] developed nested PCR for detecting NDV. Thus, in our study, we applied RT-PCR method using NDV universal primers [24] and pathotype specific primers [11]. Our result revealed that universal primers (M gene) developed by Seal et al. 1995 [24] were able to detect NDV viruses.

Pathotype specific primers developed by Kant et al. [11] were able to differentiate NDV virulence. The primers are targeting $\mathrm{F}$ gene cleavage site which has the same target sequence of forward primer (FA) and have different sequence of reverse primers (FB and FC). NDV/Ck/BGR/11, NDV/Ck/GS/14, and $\mathrm{NDV} / \mathrm{Ck} / \mathrm{LWG} / 15$ isolates were amplified using $\mathrm{NDV}-\mathrm{FA} / \mathrm{FB}$ primer, and other five isolates NDV/Ck//

\begin{tabular}{|c|c|c|c|c|c|c|c|c|c|c|c|c|c|c|c|}
\hline$\$ \mathrm{ND} / \mathrm{Ck} / \mathrm{BGR} / 11$ & GGA & GAT & $A A G$ & GCA & GIC & AAT & GIA & TAC & $\mathrm{ACC}$ & TCA TCI & CAG & $\mathrm{ACA}$ & GGG & TCA & ATC \\
\hline$\neq \mathrm{ND} / \mathrm{Ck} / \mathrm{GS} / 14$ & $\ldots$ & $\ldots \mathrm{C}$ & $\ldots$ & $\ldots$ & $\ldots$ & $\cdots$ & A. . & $\ldots$ & $\ldots$ & $\ldots G \ldots$ & $\ldots \mathrm{A}$ & $\ldots$ & $\ldots$ & $\ldots$ & $\ldots \mathrm{T}$ \\
\hline$\$ \mathrm{ND} / \mathrm{Ck} / \mathrm{JP} / 14$ & $\ldots$ & $\ldots \mathrm{C}$ & $\ldots \mathrm{A}$ & . C & $\ldots$ & $\ldots \mathrm{C}$ & A. . & $\ldots$ & $\ldots$ & $\ldots . c$ & $\ldots$ & $\ldots$ & $\ldots \mathrm{A}$ & $\ldots$ & $\ldots$ \\
\hline $\mathrm{ND} / \mathrm{Ck} / \mathrm{LG} / 15$ & $\ldots$ & $\ldots \mathrm{C}$ & $\ldots \mathrm{A}$ & . C & $\ldots$ & $\ldots \mathrm{C}$ & A. . & $\ldots$ & $\ldots$ & $\ldots . c$ & $\ldots$ & $\ldots$ & $\ldots \mathrm{A}$ & $\cdots$ & $\ldots$ \\
\hline$¥ \mathrm{ND} / \mathrm{Ck} / \mathrm{CJR} / 15$ & $\ldots$ & $\ldots \mathrm{C}$ & . A & . C & $\ldots$ & . C & A. . & $\ldots$ & $\ldots$ & $\ldots \ldots$ & $\ldots$ & $\ldots$ & $\ldots \mathrm{A}$ & $\ldots$ & $\ldots$ \\
\hline$\# \mathrm{ND} / \mathrm{Ck} / \mathrm{BGR} / 15$ & $\cdots$ & $\ldots \mathrm{C}$ & $\ldots \mathrm{A}$ & $\ldots \mathrm{C}$ & $\cdots$ & $\ldots \mathrm{C}$ & A. . & $\cdots$ & $\cdots$ & $\ldots . C$ & $\cdots$ & $\cdots$ & $\ldots \mathrm{A}$ & $\cdots$ & $\cdots$ \\
\hline$\neq \mathrm{ND} / \mathrm{Ck} / \mathrm{TRG} / 15$ & $\cdots$ & $\ldots \mathrm{C}$ & . A & $\ldots \mathrm{C}$ & $\cdots$ & $\ldots \mathrm{C}$ & A. & $\cdots$ & $\cdots$ & $\ldots . c$ & $\cdots$ & $\cdots$ & $\ldots \mathrm{A}$ & $\cdots$ & $\cdots$ \\
\hline$\neq \mathrm{ND} / \mathrm{Ck} / \mathrm{LWG} / 15$ & $\cdots$ & $\ldots \mathrm{C}$ & $\ldots \mathrm{A}$ & $\cdots$ & $\ldots$ & $\cdots$ & A. C & $\ldots$ & $\cdots$ & $\ldots G \ldots$ & $\ldots \mathrm{A}$ & $\cdots$ & $\cdots$ & $\cdots$ & $\cdots$ \\
\hline$\neq \mathrm{ND} / \mathrm{Ck} / \mathrm{BGR} / 11$ & ATA & GIC & AAG & ITG & CIC & $\operatorname{CCG}$ & AAT & ATG & $\mathrm{CCI}$ & AAG GAT & AAA & GAG & GCG & TGT & GCA \\
\hline$\$ \mathrm{ND} / \mathrm{Ck} / \mathrm{GS} / 14$ & $\ldots$ & $\ldots$ & $\ldots$ & $\ldots$ & $\ldots$ & $\ldots$ & $\ldots$ & $\ldots$ & . C & $\ldots \ldots$ & $\ldots$ & $\ldots$ & $\ldots$ & $\ldots \mathrm{C}$ & $\ldots$ \\
\hline$\$ \mathrm{ND} / \mathrm{Ck} / \mathrm{JP} / 14$ & $\ldots$ & $\ldots \mathrm{T}$ & $\ldots$ & C.C & $\ldots$ & $\ldots$ & $\ldots$ & c.. & . C & $\ldots$ & $\ldots G$ & $\ldots$ & $\ldots \mathrm{A}$ & $\ldots$ & . G \\
\hline$\$ \mathrm{ND} / \mathrm{Ck} / \mathrm{LG} / 15$ & $\ldots$ & $\ldots \mathrm{T}$ & $\ldots$ & C.C & $\ldots$ & $\ldots$ & $\ldots$ & c. . & $\ldots \mathrm{C}$ & $\ldots \ldots$ & ..G & $\ldots$ & $\ldots \mathrm{A}$ & $\ldots$ & .G \\
\hline$\$ \mathrm{ND} / \mathrm{Ck} / \mathrm{CJR} / 15$ & $\cdots$ & $\ldots \mathrm{T}$ & $\ldots$ & C.C & $\ldots$ & $\ldots$ & $\ldots$ & c. . & . C & $\ldots$ & ..G & $\ldots$ & $\ldots \mathrm{A}$ & $\ldots$ & . G \\
\hline$\neq \mathrm{ND} / \mathrm{Ck} / \mathrm{BGR} / 15$ & $\ldots$ & $\ldots \mathrm{T}$ & $\ldots$ & C.C & $\ldots$ & $\ldots$ & $\ldots$ & c.. & . C & $\ldots$ & ..G & $\ldots$ & $\ldots \mathrm{A}$ & $\ldots$ & . G \\
\hline$\$ \mathrm{ND} / \mathrm{Ck} / \mathrm{TRG} / 15$ & $\ldots$ & $\ldots T$ & $\ldots$ & C.C & $\ldots$ & $\ldots$ & $\ldots$ & c. . & $\ldots \mathrm{C}$ & $\ldots \ldots$ & $\ldots G$ & $\ldots$ & $\ldots \mathrm{A}$ & $\cdots$ & . G \\
\hline$\neq \mathrm{ND} / \mathrm{Ck} / \mathrm{LWG} / 15$ & $\cdots$ & $\cdots$ & $\cdots$ & $\cdots$ & $\cdots$ & $\cdots$ & $\cdots$ & $\cdots$ & $\ldots \mathrm{C}$ & 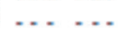 & $\cdots$ & $\cdots$ & $\cdots$ & $\ldots \mathrm{C}$ & $\cdots$ \\
\hline$\neq \mathrm{ND} / \mathrm{Ck} / \mathrm{BGR} / 11$ & AAA & GCC & $\mathrm{CCA}$ & TTA & GAG & $\mathrm{GCA}$ & TAC & AAC & AGA & ACA CTG & ACT & $\mathrm{ACC}$ & TTG & $\mathrm{CrC}$ & $\mathrm{ACT}$ \\
\hline$\$ \mathrm{ND} / \mathrm{Ck} / \mathrm{GS} / 14$ & G. & $\ldots$ & $\ldots$ & . . G & $\ldots \mathrm{A}$ & $\ldots$ & $\ldots \mathrm{T}$ & $\ldots \mathrm{T}$ & $\ldots$ & $\ldots \ldots$ & $\ldots$ & $\ldots \mathrm{A}$ & $\ldots$ & $\ldots$ & ..C \\
\hline$\$ \mathrm{ND} / \mathrm{Ck} / \mathrm{JP} / 14$ & $\ldots$ & $\ldots$ & $\ldots \mathrm{C}$ & . G & $\ldots \mathrm{T}$ & $\ldots$ & $\ldots$ & $\ldots$ & . G & $\ldots \mathrm{T} \ldots$ & $\ldots \mathrm{C}$ & $\ldots T$ & $\ldots$ & $\ldots$ & ..C \\
\hline$\$ \mathrm{ND} / \mathrm{Ck} / \mathrm{LG} / 15$ & $\ldots$ & $\ldots$ & $\ldots \mathrm{C}$ & . G & $\ldots \mathrm{T}$ & $\ldots$ & $\ldots$ & $\ldots$ & $\ldots G$ & $\ldots \mathrm{T} \ldots$ & $\ldots C$ & $\ldots \mathrm{T}$ & $\ldots$ & $\ldots$ & . C \\
\hline$\$ \mathrm{ND} / \mathrm{Ck} / \mathrm{CJR} / 15$ & $\ldots$ & $\ldots$ & $\ldots \mathrm{C}$ & . . G & $\ldots T$ & $\ldots$ & $\ldots$ & $\ldots$ & $\ldots G$ & $\ldots \mathrm{T} \ldots$ & $\ldots \mathrm{C}$ & $\ldots \mathrm{T}$ & $\ldots$ & $\ldots$ & ..C \\
\hline$\$ \mathrm{ND} / \mathrm{Ck} / \mathrm{BGR} / 15$ & $\ldots$ & $\ldots$ & $\ldots \mathrm{C}$ & . .G & $\ldots \mathrm{T}$ & $\ldots$ & $\ldots$ & $\ldots$ & $\ldots G$ & $\ldots \mathrm{T} \ldots$ & $\ldots \mathrm{C}$ & $\ldots \mathrm{T}$ & $\ldots$ & $\ldots$ & $\ldots \mathrm{C}$ \\
\hline$\# \mathrm{ND} / \mathrm{Ck} / \mathrm{TRG} / 15$ & $\cdots$ & $\ldots$ & $\ldots \mathrm{C}$ & . G & $\ldots \mathrm{T}$ & $\ldots$ & $\ldots$ & $\ldots$ & . G & $\ldots T .$. & $\ldots \mathrm{C}$ & $\ldots T$ & $\ldots$ & $\ldots$ & .CC \\
\hline$\$ \mathrm{ND} / \mathrm{Ck} / \mathrm{LWG} / 15$ & -G. & $\cdots$ & 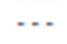 & $\ldots$ & $\ldots$ & $\cdots$ & $\ldots T$ & $\ldots T$ & . G & $\ldots \ldots$ & $\cdots$ & A & $\cdots$ & $\cdots$ & $\ldots \mathrm{C}$ \\
\hline$\$ \mathrm{ND} / \mathrm{Ck} / \mathrm{F}$ & $\operatorname{ccc}$ & CIT & $\operatorname{GgC}$ & GAI & $\mathrm{TCC}$ & ATC & CGI & APG & ATC & CAA GGG & ICG & GIG & $\mathrm{GCC}$ & $\mathrm{ACA}$ & $\mathrm{TCC}$ \\
\hline$¥ N \mathrm{ND} / \mathrm{Ck} / \mathrm{GS} / 14$ & $\ldots$ & $\ldots$ & $\ldots$ & $\ldots$ & $\ldots$ & $\ldots$ & $\ldots \mathrm{C}$ & $\ldots$ & $\ldots$ & $\ldots \ldots$ & ..C & $\ldots$ & T.. & . G & $\ldots \mathrm{T}$ \\
\hline$\$ \mathrm{ND} / \mathrm{Ck} / \mathrm{JP} / 14$ & $\ldots$ & $\ldots$ & $\ldots \mathrm{T}$ & . . C & $\ldots T$ & $\ldots$ & $\ldots$ & .G. & $\ldots \mathrm{A}$ & $\ldots$ A. & $\ldots T$ & $\ldots$ & A.T & $\ldots$ & $\ldots \mathrm{T}$ \\
\hline$\$ \mathrm{ND} / \mathrm{Ck} / \mathrm{LG} / 15$ & $\ldots$ & $\ldots$ & $\ldots \mathrm{T}$ & $\ldots \mathrm{C}$ & $\ldots T$ & $\ldots$ & $\ldots$ & -G. & $\ldots \mathrm{A}$ & $\ldots$ A. & $\ldots T$ & $\ldots$ & A.T & $\ldots$ & $\ldots T$ \\
\hline$\$ \mathrm{ND} / \mathrm{Ck} / \mathrm{CJR} / 15$ & $\ldots$ & $\ldots$ & $\ldots \mathrm{T}$ & . . C & $\ldots \mathrm{T}$ & $\ldots$ & $\ldots$ & .G. & $\ldots \mathrm{A}$ & $\ldots$ A. & $\ldots \mathrm{T}$ & $\ldots$ & A.T & $\ldots$ & $\ldots \mathrm{T}$ \\
\hline$\$ \mathrm{ND} / \mathrm{Ck} / \mathrm{BGR} / 15$ & $\ldots$ & $\ldots$ & $\ldots \mathrm{T}$ & $\ldots \mathrm{C}$ & $\ldots \mathrm{T}$ & $\ldots$ & $\ldots$ & .G. & $\ldots \mathrm{A}$ & ... A. & $\ldots \mathrm{T}$ & $\ldots$ & A.T & $\ldots$ & $\ldots T$ \\
\hline$\# \mathrm{ND} / \mathrm{Ck} / \mathrm{TRG} / 15$ & $\cdots$ & $\cdots$ & $\ldots \mathrm{T}$ & $\ldots \mathrm{C}$ & $\ldots \mathrm{T}$ & $\cdots$ & $\cdots$ & -G. & $\ldots \mathrm{A}$ & $\ldots . \mathrm{A}$. & $\ldots \mathrm{T}$ & $\ldots$ & A.T & $\cdots$ & $\ldots \mathrm{T}$ \\
\hline$\neq \mathrm{ND} / \mathrm{Ck} / \mathrm{LWG} / 15$ & $\cdots$ & . C & ... & $\cdots$ & $\cdots$ & $\cdots$ & $\ldots \mathrm{C}$ & $\ldots$ & $\cdots$ & $\ldots \ldots$ & $\ldots \mathrm{C}$ & $\ldots$ & T.. & $\ldots G$ & $\ldots \mathrm{T}$ \\
\hline$\neq \mathrm{ND} / \mathrm{Ck} / \mathrm{BGR} / 11$ & GGA & AGA & AGG & AGA & CAG & AAA & OGC & $T$ & & & & & & & \\
\hline$\$ \mathrm{ND} / \mathrm{Ck} / \mathrm{GS} / 14$ & $\cdots$ & G. . & A. & $\cdots$ & -G. & $\ldots$ & $\ldots$ & - & & & & & & & \\
\hline$+\mathrm{ND} / \mathrm{Ck} / \mathrm{JP} / 14$ & $\ldots$ & G.G & G.. & . $A G$ & $\ldots$ & GEG & $\ldots$ & C & & & & & & & \\
\hline$+\mathrm{ND} / \mathrm{Ck} / \mathrm{LG} / 15$ & $\ldots$ & G.G & G. . & .AG & $\ldots$ & GEG & $\ldots$ & C & & & & & & & \\
\hline$\# \mathrm{ND} / \mathrm{Ck} / \mathrm{CJR} / 15$ & - & G.G & G. . & $\ldots$ & $\ldots$ & GEG & $\ldots$ & C & & & & & & & \\
\hline$\neq \mathrm{ND} / \mathrm{Ck} / \mathrm{BGR} / 15$ & - & G.G & G. . & $\cdots$ & $\ldots$ & GEG & $\ldots$ & C & & & & & & & \\
\hline$\neq \mathrm{ND} / \mathrm{Ck} / \mathrm{TRG} / 15$ & $\ldots$ & G.G & G. . & . $A G$ & $\ldots$ & GEG & $\ldots$ & C & & & & & & & \\
\hline$\neq \mathrm{ND} / \mathrm{Ck} / \mathrm{LWG} / 15$ & $\ldots$ & G. . & $\ldots$ & $\ldots$ & $\ldots$ & $\cdots$ & G.. & - & & & & & & & \\
\hline
\end{tabular}

Figure-4: Partial nucleotide sequences of Newcastle disease isolates. Currently, part of F gene from position 136 to 349 is shown. Estimation of cleavage sites were marked with grey. Different nucleotide of cleavage sites among virulent isolates were marked in red font. 
$\mathrm{JP} / 14, \mathrm{NDV} / \mathrm{Ck} / \mathrm{LG} / 15, \mathrm{NDV} / \mathrm{Ck} / / \mathrm{CJR} / 15, \mathrm{NDV} / \mathrm{Ck} /$ $\mathrm{BGR} / 15$, and NDV/Ck/TRG/15 were amplified using NDV-FA/FC primer. Our result showed that both NDV virulent and avirulent strain were able to isolated from vaccinated flocks.

Nucleotide sequence of $\mathrm{F}$ gene cleavage site was used to predict pathotype of NDV [28]. A molecular basis of pathogenicity has been well established through sequence analysis of F-protein cleavage site. In general, nucleotide sequence at cleavage site of NDV virulent strains at least have three basic amino-acids (multibasic cleavage site) arginine (R) or lysine (K) in positions $112-116$ and amino acid phenylalanine (F) at position 117 , and NDV avirulent strain have less than three basic amino acids (monobasic cleavage site) in positions 112-116 and amino-acid leucine (L) at position 117 [3,14,28-30]. NDV/Ck/BGR/11 showed motif R-R-G-K-R-F which has the same motif on its $\mathrm{F}$ gene cleavage site with earlier NDV isolated in 2009-2012 in Indonesia [19]. However, NDV/Ck/LWG/15 showed motif R-R-Q-KG-F which is different from other two virulent NDV isolates. This isolat has $96 \%$ homology with NDV/Ck/ $\mathrm{GS} / 14$, however the nucleotide in position 335,341 , and 346 was different. This difference could explained based on alteration of amino acid motif. The alteration of amino acid caused by mutation or substitution was associated with the many diverse genotypes of the virus [31]. NDV/Ck/JP/14, NDV/Ck/LG/15, and NDV/Ck/TGR/15 showed motif G-K-Q-G-R-L and the NDV/Ck/JP/14 and NDV/Ck/LG/14 showed motif G-R-Q-G-R-L. Genotype I and II strains carried the fusion cleavage site motifs ${ }^{112} \mathrm{G}-\mathrm{K}-\mathrm{Q}-\mathrm{G}-\mathrm{R}-\mathrm{L}^{117}$ and ${ }^{112} \mathrm{G}-\mathrm{R}-\mathrm{Q}-\mathrm{G}-\mathrm{R}-\mathrm{L}^{117}$ [31]. The isolates were clustered in genotype I and were identical to the vaccine strain Queensland V4 and other isolates were clustered in genotype II and were very similar to the vaccine strain B1, suggesting that all genotype I and II strains were related to live vaccine strains [31]. Studies comparing the deduced amino acid sequence of the cleavage site of NDV varying in virulence for chickens shown motif ${ }^{112} \mathrm{~K} / \mathrm{R}-\mathrm{R}-\mathrm{Q} / \mathrm{R}-\mathrm{K}-\mathrm{R} / \mathrm{G}-\mathrm{F}{ }^{117}$ whereas avirulent strain of NDV show motif ${ }^{112} \mathrm{G}-\mathrm{K} / \mathrm{R}-\mathrm{Q}-\mathrm{G}-\mathrm{R}-\mathrm{L}^{117}$.

Nucleotide sequence of $\mathrm{F}$ gene cleavage site results was consistent with the result of $\mathrm{F}$ gene amplification using pathotype specific primers as presented in Figure-4, indicating that RT-PCR using pathotype specific primers NDV-FA/FB and NDV-FA/FC were able to differentiated NDV pathotype. The previous study by Wang et al. [32] and Ahmadi et al. [12] were also able to differentiate NDV pathotype using such primers. However, another study by Tiwari et al. [33] showed different phenomenon whereas NDV virulent strain were amplified using both pathotype specific primers NDV-FA/FB and NDV-FA/FC.

Development of molecular techniques such as RT-PCR offers significant advantages on laboratory diagnostic [34]. PCR technique has high sensitivity and specificity for identify pathogen, and were able to identify pathogen within species. Using this technique, we were able to do nucleotide sequence which notify difference or subtitution or mutation on species amino acid sequence. This technique were often used on molecular epidemiology study [35]. Our current study showed that NDV avirulent and virulent strain could be isolated from chickens regardless of the origin or vaccination status of the chickens. ND outbreaks among vaccinated flocks, suggesting that vaccination strategies have not effective yet in controlling the virus [36,37]. Antigenic similarity are shared among all NDV strains and these strains provides cross-protection against challenge with any other NDV strain. However, like most vaccines, NDV vaccines do not prevent vaccinated animals from becoming infected with NDV and subsequently shedding the virus [38]. Hidden immunosuppressive condition due micotoxicosis were also decreasing vaccination response [39]. Progressive genetic improvement on chicken phenotype might also increasing stress of the chicken resulting declined of immune response [40]. Matched between vaccines virus and circulating field isolates are important to provides better protection against transmission by reducing the magnitude of viral shedding. In the field, many factors play role on vaccine efficacy, thus making the antibody specificity become important. In endemic countries, aim of vaccination was not only on prevention of clinical disease and mortality, but also on decreasing the amount of virus shed from vaccinated birds [41].

Nevertheless, our findings indicates that NDV still possess threat to the poultry industries since this viruses maintained their evolution and circulation among vaccinated chickens. Some factors such stress, infection, and immunosuppressive might lead to progression of a full blown velogenic ND; If the disease progress to velogenic ND, it is going to be a potential threat to commercial poultry industry since the disease may go unnoticed and be left uncontrolled and at the end this might causing economic losses [36,37] of Indonesia's poultry industry.

\section{Conclusions}

RT-PCR and amino acid sequencing were developed for NDV detection and pathotyping. Our study showed that RT-PCR using F gene pathotype specific primers combination were able to differentiated virulent and avirulent NDV strain. Our result also provided evidence that virulent NDV strain was circulating among vaccinated flocks in West Java, Indonesia, which indicated vaccination program have not effective yet in controlling the virus, and this might possess a risk of causing ND outbreaks and causing economic losses within the poultry industry.

\section{Authors' Contributions}

DDP executed the work (collection of data, analysis, and writing of manuscript); EH participated in conception and design the study and drafting of the 
manuscript; AS participated in designed the study and drafting of the manuscript; RDS participated in designed the study, analysis of data and drafting of the manuscript, NLPIM participated in analysis and interpretation of data and drafting of the manuscript, ONP participated in analysis and interpretation of data and writing of manuscript; All authors read and approved the final manuscript.

\section{Acknowledgments}

This research was funded by Ministry of Research, Technology and Higher Education of Republic Indonesia in Hibah Bersaing Research Grant No $018.04 /$ Pl 15.8/2016.

\section{Competing Interests} interests.

The authors declare that they have no competing

\section{References}

1. Waheed, U., Siddique, M., Arshad, M., Ali, M. and Saeed, A. (2013) Preparation of new castle disease vaccine from VG/ GA strain and its evaluation in commercial broiler chicks. Pak. J. Zool., 45(2): 339-344.

2. Miller, P.J. and Guus, K. (2013) Newcastle disease. In: Swayne, D.E., Glisson, J.R., McDougald, L.R., Nolan, L.K., Suarez, D.L. and Nair, V., editors. Diseases of Poultry. $13^{\text {th }}$ ed. Wiley-Blackwell, Ames, IA. p98-107.

3. Office International des Epizooties (OIE). (2012) Newcastle disease. Infection with Newcastle Disease Virus. OIE, Paris. p555-574.

4. Aldous, E.W. and Alexander, D.J. (2001) Detection and differentiation of newcastle disease virus (avian paramixovirus Type 1). Avian Pathol., 30: 117-128.

5. Boynukara, B., Gulhan, T., Coven, F., Kiziroglu, I. and Durmus, A. (2013) Determination of Newcastle disease virus among wild bird populations in Lake Van basin, Turkey. Turk. J. Vet. Anim. Sci., 37: 1-9.

6. Alexander, D.J. and Jones, R.C. (2000) Paramyxoviridae. Newcastle disease virus and other avian paramixoviruses. Rev. Sci. Tech., 19(2): 443-462.

7. Nanthakumar, T., Katari, R.S., Tieari, A.K., Butchaiah, G. and Kataria, J.M. (2000) Pathotyping of newcastle disease viruses by RT-PCR and restriction enzyme analysis. Vet. Res. Commun., 24: 275-286.

8. Cattoli, G., Susta, L., Terregino, C. and Brown, C. (2011) Newcastle disease: A review of field recognition and current methods of laboratory detection. J. Vet. Diagn. Invest., 23: 637-656

9. Viljoen, G.J., Nel, L.H. and Crowther, J.R., (2005) Molecular Diagnostic PCR Handbook. Springer, Dordrecht.

10. Panda, A., Huang, Z., Elankumaran, S., Rockemann, D.D. and Samal, S.K. (2004) Role of fusion protein cleavage site in the virulence of Newcastle disease virus. Microb. Pathog., 36: 1-10.

11. Kant, A., Koch, G., Roozelaar, F., Balk, F. and Huurne, A.T. (1997) Differentiation of virulent and non-virulent strains of Newcastle disease virus within 24 hours by polymerase chain reaction. Avian Dis., 26: 837-840.

12. Ahmadi, E., Pourbakhsh, S.A., Ahmadi, M. and Talebi, A. (2014) Pathotypic characterization of Newcastle disease virus isolated from commercial poultry in Northwest Iran. Turk. J. Vet. Anim. Sci., 38: 383-387.

13. Yu, L., Wang, Z., Jiang, Y., Chang, L. and Kwang, J. (2001) Characterization of newly emerging Newcastle disease virus isolates from the People's Republic of China and Taiwan. J. Clin. Microbiol., 39: 3512-3519.

14. Alexander, D.J. (2009) Ecology and epidemiology of
Newcastle disease. In: Capua, I. and Alexander, D.J., editor. Avian Influenza and Newcastle Disease. Springer, Milan. p19-26.

15. Madadgar, O., Karimi, V., Nazaktabar, A., Kazemimanesh, M., Ghafari, M.M., Dezfouli, S.M.A. and Hojjati, P. (2013) A study of Newcastle disease virus obtained from exotic caged birds in Tehran between 2009 and 2010. Avian Pathol., 42(1): 27-31.

16. Director General of Livestock Services (DGLS). (2011) Statistical on Livestock 2011. Ministry of Agriculture, Indonesia.

17. Dharmayanti, N.L.P., Hartawan, R., Hewajuli, D. and Indriani, R. (2014) Phylogenetic analysis on Genotype VII of Newcastle disease virus in Indonesia. Afr. J. Microbiol. Res., 8(13): 1368-1374.

18. Xiao, S., Paldurai, A., Nayak, B., Samuel, A., Bharoto, E.E., Prajitno, T.Y., Collins, P.L. and Samal, S.K. (2012) Complete genome sequences of Newcastle disease virus strains circulating in chicken populations of Indonesia. J. Virol., 86(10): 5969-5970.

19. Miller, P.J., Haddas, R., Simanov, L., Lublin, A., Rehmani, S.F., Wajid, A., Bibi, T., Khan, T.A., Yaqub, T., Setiyaningsih, S. and Afonso, C.L. (2015) Identification of new sub-genotype of virulent Newcastle disease virus with potential panzootic feature. J. Infect. Genet. Evol., 29: 216-229.

20. Qiagen, QIAamp ${ }^{\circledR}$ Viral RNA Mini Handbook. For Purification of Viral RNA from Plasma, Serum, CellFree Body Fluids, and Cell-Culture Supernatants. $4^{\text {th }}$ ed. December, 2014. Available from: https://www.qiagen.com/ be/resources/resourcedetail?id $=\mathrm{c} 80685 \mathrm{c} 0-4103-49$ ea-aa728989420e3018\&lang=en. Accessed on 15-01-2015.

21. Tamura, K., Stecher, G., Peterson, D., Filipski, A. and Kumar, S. (2013) MEGA6: Molecular evolutionary genetics analysis version 6.0. Mol. Biol. Evol., 30(12): 2725-2729.

22. Jestin, V. and Jestin, A. (1991). Detection of Newcastle disease virus RNA in infected allantoic fluids by in vitro enzymatic amplification (PCR). Arch. Virol., 118(3): 151-161.

23. Kho, C.L., Mohd Azmi, M.L., Arshad, S.S. and Yusoff, K. (2000) Performance of an RT-nested PCR ELISA for detection of Newcastle disease virus. J. Virol. Methods, 86: 71-83.

24. Seal, B.R., King, D.J. and Bennett, J.D. (1995) Characterization of Newcastle disease virus isolates by reverse transcription PCR coupled to direct nucleotide sequencing and development of sequence database for pathotype prediction and molecular epidemiological analysis. J. Clin. Microbiol., 33: 2624-2630.

25. Rabalski, L., Smietanka, K., Minta, Z. and Szewczyk, B. (2014) Detection of Newcastle disease virus minor genetic variants by modified single-stranded conformational polymorphism analysis. BioMed Res. Int., 2014: 8

26. Lai, K.S., Yusoff, K. and Maziha, M. (2012) Heterologous expression of hemagglutinin-neuraminidase protein from Newcastle disease virus strain AF2240 in Centella asiatica. Acta Biol. Cravov. Bot., 54(1): 142-147.

27. Al-Shammari, A.M., Al-Nassrawei, H.A. and Murtadha, A.M.H. (2014) Molecular diagnosis of Newcastle disease Iraqi Virulent strain virus HN gene by specific primers design. Vet. Med. Sci., 5(2): 196-203.

28. Vidanovic, D., Sekler, M., Polacek, V., Vaskovic, N., Asanin, R., Milic, N. and Nisavic, J. (2012) Characterization of Newcastle disease virus and poultry-handling practices in live poultry markets, Ethiopia. Application of standard and molecular methods for the diagnosis of Newcastle disease. Arch. Biol. Sci., 64(4): 1433-1437.

29. Wen, G., Shang, Y., Guo, J., Chen, C., Shao, H., Luo, Q., Yang, J., Wang, H. and Cheng, G. (2013) Complete genome sequence and molecular characterization of thermostable Newcastle disease virus strain TS09-C. Virus Genes, 46: 542-545.

30. Farooq, M., Saliha, U., Munir, M. and Khan, Q.M. (2014) 
Biological and genotypic characterization of the Newcastle disease virus isolated from disease outbreaks in commercial poultry farms in Northern Punjab, Pakistan. Virol. Rep., 3: 30-39.

31. Snoeck, C.J., Owoade, A.A., Couacy-Hymann, E., Alkali, B.R., Okwen, M.P., Adeyanju, A.T., Komoyo, G.F., Nakoun, E., Faou, A.L. and Muller, A.P. (2013) High genetic diversity of Newcastle disease virus in poultry in West and Central Africa: Co-circulation of genotype XIV and newly defined genotypes XVII and XVIII. J. Clin. Microbiol., 51(7): 2250-2260

32. Wang, Z., Vreede, F.T., Mitchell, J.O. and Viljoen, G.J. (2001) Rapid detection and differentiation of Newcastle disease virus isolates by a triple one-step RT-PCR. Onderstepoort J. Vet. Res., 68(2): 131-134.

33. Tiwari, A.K., Kataria, R.S., Nanthakumar, T., Dash, B.B. and Desai, G. (2004) Differentiation detection of Newcastle disease virus strains by degenerate primers based RT-PCR. Comp. Immunol. Microbiol. Infect. Dis., 27: 163-169.

34. Haque, M.H., Hossain, M.T., Islam, M.T., Zinnah, M.A., Khan, M.S.R. and Islam, M.A. (2010) Isolation and detection of Newcastle disease virus from field outbreaks in broiler and layer chickens by reverse transcription-polymerase chain reaction. Bangladesh J. Vet. Med., 8(2): 87-92.

35. Rehmani, S.F., Wajid, A., Bibi, T., Nasir, B., Mukhtar, N., Hussain, A., Lone, N.A., Yaqub, T. and Afonso, C.L. (2015) Presence of virulent Newcastle disease virus in vaccinated chickens in farms in Pakistan. J. Clin. Microbiol., 53(5): 1715-1718.

36. Munir, M., Zohari, S., Abbas, M. and Berg, M. (2012)
Sequencing and analysis of the complete genome of Newcastle disease virus isolated from a commercial poultry farm in 2010. Arch. Virol., 157: 765-768.

37. Shabbir, M.Z., Zohari, S., Yaqub, T., Nazir, J., Shabbir, M.A.B., Shafee, M., Sajid, M., Anees, M., Abbas, M., Khan, M.T., Ali, A.A., Ghafoor, A., Ahad, A., Channa, A.A., Anjum, A.A., Hussain, N., Ahmad, A., Goraya, M.U., Iqbal, A., Khan, S.A., Aslam, H., Zehra, K., Sohail, M.U., Yaqub, W., Ahmad, N., Berg, M. and Munir, M. (2013) Genetic diversity of Newcastle disease virus in Pakistan: A countrywide perspective. Virol. J., 10: 170.

38. Chukwudi, O.E., Chukwuemeka, E.D. and Mary, U. (2012) Newcastle disease virus shedding among healthy commercial chickens and its epidemiological importance. Pak. Vet. J., 32(3): 354-356.

39. Henning, J., Morton, J., Hla, T. and Meers, J. (2008) Mortality rates adjusted for unobserved deaths and associations with Newcastle disease virus serology among unvaccinated village chickens in Myanmar. Prev. Vet. Med., 85: 241-252.

40. Hsiang-Jung, T., Kuo-Hui, C., Chun-Hsien, T., Manvell, R.J. and Alexander, D.J. (2004) Antigenic and genotypical characterization of Newcastle disease viruses isolated in Taiwan between 1969 and 1996. Vet. Microbiol., 104: 19-30.

41. Miller, P.J., Afonso, C.L., Atteche, J.E., Dorsey, K.M., Courtney, S.C., Guo, Z. and Kapczinski, D.R. (2013) Effects of Newcastle disease virus vaccine antibodies on the shedding and transmission of challenge viruses. Dev. Comp. Immunol., 41(4): 505-513. 